\title{
Effect of simvastatin on thrombopoiesis in porcine bone marrow
}

\author{
Anna Snarska, Dominika Wysocka, Liliana Rytel \\ Department and Clinic of Internal Diseases, Faculty of Veterinary Medicine, \\ University of Warmia and Mazury, 10-719 Olsztyn, Poland \\ anna.snarska@uwm.edu.pl
}

Received: August 12, $2018 \quad$ Accepted: February 12, 2019

\begin{abstract}
Introduction: Statins are pharmacological agents commonly used to lower serum cholesterol level. The aim of the experiment was to investigate the effect of the statin simvastatin on thrombopoiesis in the porcine model because it is the closest to the human one regarding physiological and genetic similarities. Material and Methods: The study was conducted on a group of 32 pigs randomly divided into two equal groups: control and experimental. The pigs were treated for 28 and 56 days with simvastatin in a dose of $40 \mathrm{mg}$ per day per animal. Cytological evaluation of bone marrow smears was performed to assess the average number of all types of cells during thrombopoiesis as was analysis of haematological parameters to assess PLT and MPV. Results: During the course of the experiment statistically significant changes in the number of promegakaryocytes were observed. Other parameters also showed some fluctuations during the study. However, these changes were not statistically significant. Conclusion: The obtained results clearly indicate a toxic influence of simvastatin on the process of thrombopoiesis and prove that statins reduce mean platelet volume, thus affecting the process of clot formation through the period of administration in a duration-dependent manner.
\end{abstract}

Keywords: swine, simvastatin, bone marrow, haematopoiesis, thrombopoiesis.

\section{Introduction}

Simvastatin is the best-known statin used in humans and animals to reduce serum levels of total cholesterol (TC) and low-density lipoprotein (LDL). It is a natural metabolite of Aspergillus terreus. Simvastatin is administered in an inactive form, which is activated in the liver into the active form of hydroxy acid. The mechanism of action of statins is a reversible inhibition of 3-hydroxy-3-methylglutarylcoenzyme A reductase (HMG-CoA reductase) - the most important enzyme in cholesterol biosynthesis. Simvastatin is eliminated by the kidneys at a low rate and therefore can be used in patients with renal failure. Liver diseases occurring with high levels of aminotransferases, pregnancy, lactation, and muscle diseases are an absolute contraindication to the use of simvastatin. In dogs, statins, most commonly simvastatin, are successfully used to treat disorders of lipid metabolism manifested by hyperlipoproteinemia (7). Studies in rodents clearly indicate that statins can initiate osteolytic processes by initiating differentiation of osteoblasts (12). Statins lower the normal and elevated levels of total cholesterol and its serum fractions very, effectively and dose-dependently. An increase in the high density lipoprotein (HDL) fraction and a decrease in triglyceride (TG) concentration are undoubtedly beneficial for patients during statin therapy. It is also assumed that statins exhibit neuroprotective effects on the central nervous system through pleiotropic effects, including antioxidant, anti-inflammatory, antithrombotic, immunomodulatory, and stabilising atherosclerotic plaques (1).

Studies in rats and dogs show that statins (particularly lovastatin) have a strong teratogenic effect, and the presence of cataracts has been observed in dogs during the course of treatment using these compounds (2). Most statin side effects are mild and transient and include: muscular weakness, muscle fatigue, visual and taste disturbances, and gastrointestinal disorders. The most serious side effects in humans and animals are movement disorders associated with the occurrence of myopathy with significant pain and severe muscle weakness. Studies conducted on humans indicate an increased risk of bone fractures and disturbances in bone 
metabolism in the course of statin therapy $(4,5)$. In some cases, the occurrence of anaemia (21), a decrease in the platelet count (PLT), and a slight degree of leukocytosis caused by an increase in the number of eosinophils were observed in the course of statin therapy (11).

Statins are drugs most commonly used worldwide to reduce the activity of platelets in processes of clot formation in patients with hypercholesterolemia $(17,18)$. Platelets play a key role in thrombogenesis and are actively involved in the progression of atherosclerotic lesions. The use of statins in human medicine effects a significant change in mean platelet volume (MPV), which affects the weakening of clot formation (21). MPV has recently become an interesting topic in our research because the effect of simvastatin on haematopoietic processes is not fully understood. In addition, statins significantly increase the activity of monocytes and macrophages, increasing the risk of thrombosis in the course of atherosclerosis (16). In this experiment, the porcine model was used, which is the closest to the human one regarding physiological and genetic similarities.

The aim of this study was to determine whether simvastatin demonstrates a toxic effect on the process of thrombopoiesis displayed in reduction of the number of platelets and the mean platelet volume.

\section{Material and Methods}

The study was conducted on 32 clinically healthy Large White Polish gilts with a body weight of 35$40 \mathrm{~kg}$. During the experiment, the animals were kept in standard laboratory conditions and fed a commercial diet appropriate for the age and species. Animals were randomly divided into two equal groups: control (C) and experimental $(\mathrm{E})$. In the experimental group simvastatin at a dose of $40 \mathrm{mg}$ was administered orally for 56 days (as one tablet once a day). At the same time, a placebo was administered to the control animals (as one empty gelatine capsule once a day). Three bone marrow samples were taken from all animals: on day 0 (a day before beginning simvastatin administration), and on the $28^{\text {th }}$, and the $56^{\text {th }}$ days of the experiment. Bone marrow was sampled from the lateral condyle of the femur under local anaesthesia with xylazine hydrochloride (Rompun, Bayer, Germany, $1.5 \mathrm{mg} / \mathrm{kg}$ b.w., intramuscularly), and zolazepam with tiletamine (Zoletil, Virbac, France, $2.2 \mathrm{mg} / \mathrm{kg}$ of body weight, intramuscularly), using biopsy needles (Jamshidi, DePuy Synthes, Austria). After collection, bone marrow was used immediately to perform bone marrow smears. Smears were stained with the May-Grünwald-Giemsa method (May-Grünwald stain for 2 min and Giemsa stain diluted with 9 volumes

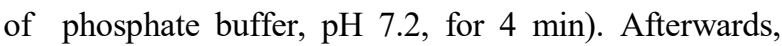
smears were evaluated under a light microscope (Eclipse 80i, Nikon, Japan) using an SH-96/24D haematological counter (Alchem, Poland). The numbers of particular forms of cells in the platelet line of the bone marrow - megakaryoblasts, promegakaryocytes, and megakaryocytes - were defined in relation to 1,000 of all bone marrow cells. Statistical analysis was performed with an ANOVA test using Statistica 10 software (StatSoft, now Tibco, USA) after checking the assumptions required for ANOVA. The differences were considered statistically significant at $\mathrm{P} \leq 0.05$. In parallel to bone marrow sampling, peripheral blood was collected into $2 \mathrm{~mL}$ test tubes with $\mathrm{K}_{2}$ EDTA (Vacuette, Greiner Bio-One, Austria). Platelet morphology was determined using a Siemens ADVIA 2120i diagnostic haematology analyser (USA). PLT and MPV were analysed. The obtained haematological results were also subjected to statistical analysis (ANOVA test).

\section{Results}

The results of this study are presented in tabular form (Tables 1 and 2) and supplemented with figures (Figs 1-3). There were no significant differences in the number of platelets in whole blood between the control and experimental groups at the beginning of the experiment (day 0).

During the first half of the course of the study, a decrease in the number of platelets from 377.06 to 364.75 (Table 1) was observed in the experimental group, as seen on the $28^{\text {th }}$ day. In addition, the average MPV in this period was lower at 6.49 (fl).

The number of platelets in tested whole blood was only slightly lower over the second half of the study than on day 0 and was recorded at $376\left(10^{3} / \mu \mathrm{L}\right)$ on the $56^{\text {th }}$ day of the study. The average MPV at that time was 6.48 (fl). Differences in the values of these parameters were not significant.

During the entire experiment, the average number of platelets and their volume were higher in the control group. However, significant differences between control and experimental group were not observed.

Cytological evaluation of bone marrow smears of experimental animals on day 28 revealed an increase in the number of megakaryoblasts and promegakaryocytes (Fig. 1) from 0.187 to 0.193 and 0.105 to 0.123 , respectively (Table 2). At the same time, a decrease in the number of megakaryocytes from 0.731 to 0.706 was observed.

The number of megakaryocytes (Fig. 2) in the experimental group was slightly higher than in the control group (Fig. 3) and reached 0.718 on the $56^{\text {th }}$ day of the study. The average number of megakaryoblasts and promegakaryocytes declined 0.181 and 0.104, respectively.

Over the whole experiment, the number of promegakaryocytes showed statistically significant differences between groups and samplings $(\mathrm{P}<0.05)$. Other types of cells did not return statistically significant differences in their counts. 
Table 1. Selected parameters of whole blood (mean \pm SD)

\begin{tabular}{llll}
\hline & Day & Control & Experimental \\
\hline \multirow{2}{*}{$\begin{array}{l}\text { PLT }\left(10^{3} / \mu \mathrm{L}\right) \\
\text { Total platelets }\end{array}$} & 0 & $384.94 \pm 59.21$ & $377.06 \pm 78.41$ \\
\cline { 2 - 4 } & 28 & $380.25 \pm 56.51$ & $364.75 \pm 63.29$ \\
\cline { 2 - 4 } & 56 & $379.19 \pm 56.57$ & $376.00 \pm 75.94$ \\
\hline \multirow{2}{*}{$\begin{array}{l}\text { MPV (fL) } \\
\text { Mean platelet volume }\end{array}$} & 0 & $7.52 \pm 1.40$ & $6.61 \pm 0.31$ \\
\cline { 2 - 4 } & 28 & $7.58 \pm 1.49$ & $6.49 \pm 0.40$ \\
\hline
\end{tabular}

Statistically significant differences between groups $(\mathrm{P}<0.05)$ are marked by capital letters

Table 2. The average number of thrombopoietic cells for three lines in relation to 1,000 of all porcine bone marrow cells (mean \pm SD)

\begin{tabular}{llll}
\hline & Day & Control & Experimental \\
\hline \multirow{3}{*}{ Megakaryoblasts } & 0 & $0.268 \pm 0.101$ & $0.187 \pm 0.135$ \\
\cline { 2 - 4 } & 28 & $0.268 \pm 0.149$ & $0.193 \pm 0.138$ \\
\cline { 2 - 4 } Promegakaryocytes & 56 & $0.237 \pm 0.120$ & $0.181 \pm 0.115$ \\
\hline \multirow{3}{*}{ Megakaryocytes } & $0.168 \pm 0.087^{\mathrm{A}}$ & $0.105 \pm 0.026^{\mathrm{Ba}}$ \\
\cline { 2 - 4 } & 28 & $0.150 \pm 0.063^{\mathrm{A}}$ & $0.123 \pm 0.078^{\mathrm{Bab}}$ \\
\hline & 56 & $0.131 \pm 0.060^{\mathrm{A}}$ & $0.104 \pm 0.025^{\mathrm{Bab}}$ \\
\hline & 28 & $1.068 \pm 0.233$ & $0.731 \pm 0.315$ \\
\hline & 56 & $1.087 \pm 0.303$ & $0.706 \pm 0.319$ \\
\hline
\end{tabular}

Statistically significant differences between samplings $(\mathrm{P}<0.05)$ are marked by small letters ${ }^{\mathrm{a}}$ - difference between day 0 and day $28,{ }^{\mathrm{b}}$ - difference between days 28 and 56)

Statistically significant differences between groups $(\mathrm{P}<0.05)$ are marked by capital letters
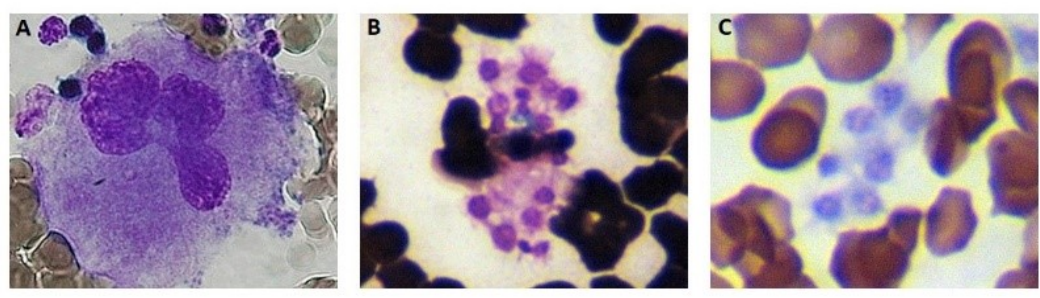

Fig. 1. Experimental group - day 28. A - megakaryocyte, B-C - platelet aggregate
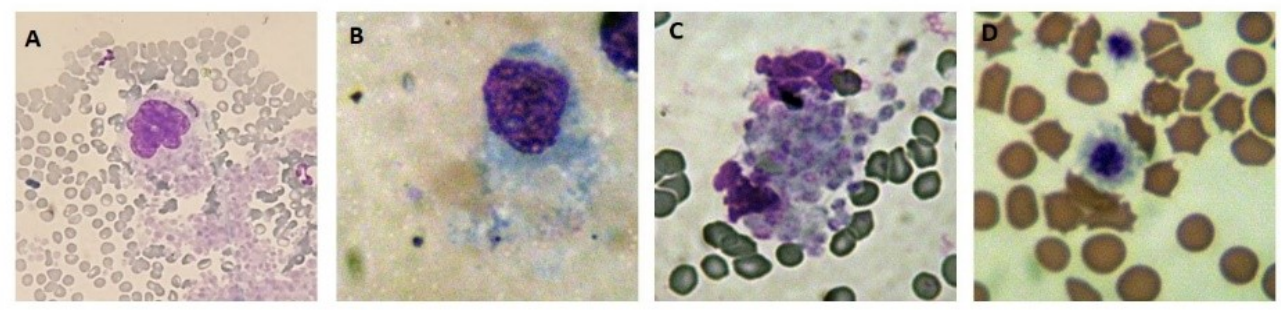

Fig. 2. Experimental group - day 56. A - megakaryoblast, B - promegakaryocyte, C - megakaryocyte, D - platelets

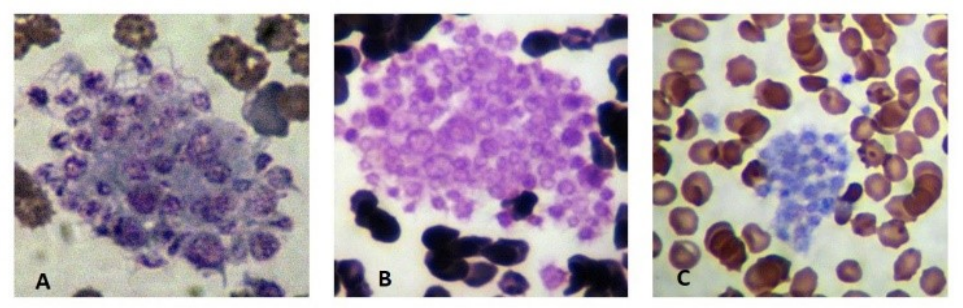

Fig. 3. Control group. A - megakaryocyte, B-C - platelets 


\section{Discussion}

The main finding of our study is that the use of simvastatin in a dose of $40 \mathrm{mg} /$ day per animal results in a significant decrease in the number of promegakaryocytes in bone marrow smears, which may suggest a slight weakening of haematopoietic processes. It is worth emphasising that the number of promegakaryocytes in the experimental group was the lowest on day 56 at the end of the study.

The anti-thrombotic effects of statins are known and have been documented in many investigations (14, 19). The study by Gaddam et al. (6) showed how greatly statins affect thrombocyte adhesion abilities, and hence the weakening of the activation of clot formation. A study in patients with hyperlipidaemia proved that the use of statins significantly weakens platelet aggregation and slightly lowers fibrinogen concentration (20).

Platelets are traditionally recognised for their central role in haemostasis. However, many lines of research clearly demonstrate that they are potent immune modulators and effectors (8). The processes of platelet activation and aggregation play a key role in the pathophysiology of cardiovascular diseases (23). The results obtained by the authors of the research referred to above prove that simvastatin increased the efficiency of the mesenchymal stem cells by reducing inflammation and oxidative stress, thus demonstrating definite beneficial effects for the patient. Chello et al. (3) prove that simvastatin increases apoptosis of neutrophils and reduces inflammatory reactions after surgical procedures in the myocardium. The advantageous effect of statins in cardiac patients is based on prevention of clot formation, but it might be harmful to other patients due to an increased risk of haemorrhage.

However, knowledge of the influence of statins on the process of haematopoiesis in bone marrow is less advanced. In our study, the influence of simvastatin on haematopoiesis was visible on the $28^{\text {th }}$ day, which was demonstrated in an increase in the number of megakaryoblasts and promegakaryocytes, and in a decrease in megakaryocytes. At that time, the number of platelets in the experimental group was the lowest. Studies carried out by Newman et al. (13) indicated that in the treatment of oncological patients statins had a beneficial protective effect on bone marrow cells during aggressive chemotherapy. Statins may increase erythropoiesis by mechanisms independent of erythropoietin (22) and cause the activation of the leukocytic system.

Assessment of haematological parameters demonstrated that simvastatin slightly decreases PLT and reduces MPV. MPV is a simple measure of platelet activation. A rise in the platelet volume is connected with the change in platelet shape from discoid to spherical and swollen $(9,24)$. The study by Martin et al. (10) proves that the activation of platelets consists, among other things, in a gain in their volume, so that they adhere to vessels and form plate aggregates more easily than platelets with a lower volume. Our study clearly indicates a reduction in the number of platelets on the $28^{\text {th }}$ day of simvastatin administration. Also, the average platelet volume shrank and reached the lowest value on day 56. However, these differences were not statistically significant.

Pucetti et al. (16) demonstrated that platelets enhanced monocyte production of tissue factor, and statins were able to counteract this prothrombotic mechanism. Motawi et al. (11) showed that simvastatin has therapeutic and protective effects on bone marrow cells in the course of liver fibrosis in rats. A study by Pagkalos et al. (15) shows that high concentrations of simvastatin may have a toxic effect on bone marrow cells, but interestingly that they also caused a beneficial mineralisation effect in bone tissue.

The results obtained during the present investigation clearly show that simvastatin suppresses haematopoiesis, which is manifested by the decrease in the number of platelets in whole blood and promegakaryocytes in bone marrow. Therefore, it can be concluded that the use of simvastatin may weaken the platelet-forming activity of the bone marrow at the stage of promegakaryocytes.

Conflict of Interests Statement: The authors declare that there is no conflict of interests regarding the publication of this article.

Financial Disclosure Statement: The authors declare that they did not receive any financial support. It was a self-funded study.

Animal Rights Statement: The present study was conducted according to the guidelines of the Local Ethical Committee in Olsztyn (Poland), (Decision No. $61 / 2010 \mathrm{~W}$ of 17 June 2010).

\section{References}

1. Antons K.A., Williams C.D., Baker S.K., Philips P.S.: Clinical perspectives of statin-induced rhabdomyolysis. Am J Med 2006, 119, 400-409.

2. Black A.E., Hayes R.N., Roth B.D., Woo P., Woolf T.F.: Metabolism and excretion of atorvastatin in rats and dogs. Drug Metab Dispos 2000, 27, 916-992.

3. Chello M., Anselmi A., Spadaccio C., Patti G., Goffredo C., Di Sciascio G., Covino E.: Simvastatin increases neutrophil apoptosis and reduces inflammatory reaction after coronary surgery. Ann Thorac Surg 2007, 83, 1374-1380.

4. Cui Q., Wang G.J., Su C.C., Balian G.: The Otto Aufranc Award: lovastatin prevents steroid induced adipogenesis and osteonecrosis. Clin Orthop 1997, 344, 8-19.

5. Edwards C.J., Hart D.J., Spector T.D.: Oral statin and increased bone mineral density in postmenopausal women. Lancet 2000, $355,2218-2219$

6. Gaddam V., Li D.Y., Mehta J.L.: Anti-thrombotic effects of atrovastatin- an effect unrelated to lipid lowering. J Cardiovasc Pharmacol Ther 2002, 7, 247-253. 
7. Glińska K., Nicpoń J., Pawlas M.: Mechanism of action of statins and possibilities of their application in dog therapy. Med Weter 2006, 62, 254-256.

8. Jenne C.N., Kubes P.: Platelets in inflammation and infection. Platelets 2015, 26, 286-292.

9. Martin J.F., Bath P.M.W., Burr M.L.: Influence of platelet size on outcome after myocardial infarction. Lancet 1991, 338, 1409-1411.

10. Martin J.F., Trowbridge E.A., Salmon G., Plumb J..: The biological significance of platelet volume: its relationship to bleeding time, thromboxane B2 production and megakaryocyte nuclear DNA concentration. Thromb Res 1983, 32, 443-460.

11. Motawi T.M., Atta H.M., Sadik N.A., Azzam M..: The therapeutic effects of bone marrow-derived mesenchymal stem cells and simvastatin in a rat model of liver fibrosis. Cell Biochem Biophys 2014, 68, 111-25.

12. Mundy G., Garrett R., Harris S., Chan J., Chen D., Rossini G., Boyce B., Zhao M., Gutierrez G.: Stimulation of bone formation in vitro and in rodents by statins. Science 1999, 286, 1946-1949.

13. Newman A., Clutterbuck R.D., Powles R.L., Chan J., Chen D., Rossini G., Boyce B., Zhao M., Gutierrez G.: Selective inhibition of primary acute myeloid leukaemia cell growth by simvastatin. Leukemia 1994, 8, 2023-2029.

14. Owens A.P. $3^{\text {rd }}$, Mackman N.: The antithrombotic effects of statins. Annu Rev Med 2014, 65, 433-445.

15. Pagkalos J., Cha J.M., Kang Y., Heliotis M., Tsiridis E., Mantalaris A.: Simvastatin induces osteogenic differentiation of murine embryonic stem cells. J Bone Miner Res 2010, 25, 2470-2478.

16. Puccetti L., Bruni F., Bova G., Cercignani M., Pompella G., Auteri A., Pasqui A.L.: Role of platelets in tissue factor expression by monocytes in normal and hypercholesterolaemia subjects. In vivo effect of cerivastatin. Int J Clin Lab Res 2000 , 30, 147-156.

17. Puccetti L., Pasqui A.L., Auteri A., Bruni F.: Mechanisms for antiplatelet action of statins. Curr Drug Targets Cardiovasc Haematol Disord 2005, 5, 121-126.

18. Puccetti L., Pasqui A.L., Pastorelli M., Bova G., Cercignani M., Palazzuoli A., Angori P., Auteri A., Bruni F.: Time-dependent effect of statins on platelet function in hypercholesterolaemia. Eur J Clin Invest 2002, 32, 901-908.

19. Rodriguez A.L., Wójcik B.M., Wróbleski S.K., Myers D.D. Jr, Wakefield T.W., Diaz J.A.: Statins, inflammation and deep vein thrombosis: a systematic review. J Thromb Thrombolysis 2012, 33, 371-382.

20. Sikora J., Kostka B., Marczyk I., Krajewska U., Chałubiński M., Broncel M.: Effect of statins on platelet function in patients with hyperlipidemia. Arch Med Sci 2013, 9, 622-628.

21. Sivri N., Tekin G., Yalta K., Aksoy Y., Senen K., Yetkin E.: Statins decrease mean platelet volume irrespective of cholesterol lowering effect. Kardiol Pol 2013, 71, 1042-1047.

22. Snarska A., Gonkowski S., Rytel L., Pomianowski A., Babińska I., Otrocka-Domagała I., Żarczyńska K., Wysocka D., Sobiech P.: The influence of simvastatin on red blood cells line in the porcine bone marrow. Pol J Vet Sci 2017, 20, 743-746.

23. Yang Y., Qian H., Huang J., Li J.J., Gao R.L., Dou K.F., Yang G.S., Willerson J.T., Geng Y.J.: Combined therapy with simvastatin and bone marrow-derived mesenchymal stem cells increases benefits in infarcted swine hearts Arterioscler Thromb Vasc Biol 2009, 29, 2076-2082.

24. Yetkin E.: Mean platelet volume not so far from being a routine diagnostic and prognostic measurement. Thromb Haemost 2008, $100,3-4$. 